\title{
On Equal Terms? On Implementing Infants' Cultural Rights
}

\author{
Pauline von Bonsdorff
}

\begin{abstract}
How can we implement infants' cultural rights? Is there even reason to confer such rights to non-speaking children, or is it enough that we recognise slightly older children as culturally active individuals? Acknowledging children's intellectual capacities and their right to be heard in matters that concern them are important threads in research on children and ideals of childrearing during the last hundred years. This development is parallel with the one leading from the Declaration of the Rights of the Child in 1923 to the UN Convention on the Rights of the Child in 1989. The spirit of human rights that informs these documents cannot be underestimated. Yet reading the Convention carefully one observes that infants, literally "non-speakers", are challenging in the discourse of human rights, which emphasises speech and language. What is an infant, then? While non-speakers, infants are highly social and communicative, using their whole body in multimodal, active and responsive gestures. This is often overlooked in both research and practices, as I show in my chapter. Instead of noticing the similarities between infants and adults, infants still tend to be represented as different and "other", as compared to the adult. I suggests that we need a more holistic approach, which does justice to infants' playful, interactive and affectionate initiatives. We need to be sensitive not just to what is generalizable, but also to particular contexts, situations and cultures of interaction. This way it might be possible to better acknowledge and cater for infants' cultural rights.
\end{abstract}

\section{Keywords}

children's cultural rights - infant communication - infant aesthetics - play imitation theory - copycat babies 
I held a baby who wasjust under two weeks old and looked at her. She seemed to look back, although due to her dark blue eyes I could not see exactly where she was focusing. I showed my tongue. After a short while, she showed hers. I put my tongue in one corner of the mouth. She responded with the same gesture, and we went on for a short while. A few days later, as I held her again in a similar situation, she initiated the game by showing her tongue. And I showed mine. ${ }^{1}$

According to empirical research and theoretical and methodological arguments especially from childhood studies (James, Jenks, \& Prout, 1998; Corsaro, 2018) we should consider children as agents. From the point of view of culture, this means they are persons capable of contributing to culture in their families and communities. The development of this view in the research community is parallel with the implementation of the United Nations Convention on the Rights of the Child from 1989 and is part of the same intellectual current. ${ }^{2}$ Moreover, research about infants' cognitive and communicative capacities from the late 2oth and early 21st centuries has brought about a recognition of the continuities in human development from infancy through to childhood and adulthood (Gopnik, 2009; Reddy, 2008; Stern, 2010). Due to this body of research, we can now acknowledge that infants are similar to older children and adults in many respects rather than just different, even categorically, from them.

In terms of cultural rights, infants however pose a challenge to the ethos and formulations of the Convention. This challenge is related to their condition as individuals who do not speak and to a slight bias, in the text of the Convention, towards the normative idea that human beings are autonomous and rational. The child, as it appears in the text, is primarily a speaking child, as implied by locutions such as "freedom of expression" (article 13:1) and "freedom of thought" (article 14:1). ${ }^{3}$ There is no mention of communication and interaction as processes where we influence each other and create meaning together. Rather, children are either speaking subjects who voice their thoughts and express themselves, or subject to education (article 29). ${ }^{4}$ This creates a lacuna with regard to implementing especially infants', but also older children's cultural rights. Yet especially in infancy, intersubjectivity and interdependence are paramount, and communication is highly reciprocal and embodied (Malloch \& Trevarthen, 2009a).

The Convention might be symptomatic of an "adultocentric" (Kennedy, 2006, p. 67) view of human beings; one that suits the rational adult but is less 
fitting for children, let alone non-speaking infants. In this Chapter I discuss, in a partly constructive and partly critical mode, how we might think about and act with infants in ways that make more justice to them as cultural beings that are both similar to and different from older people. I start by outlining an "existential" approach that recognises the embodied, relational, and affective dimensions of infant agency and takes into account the situation and context of interactions. I then turn to cognitivism in child psychology, more precisely to the "imitation theory" of new-borns' responses to facial gestures. My conclusion is that the term imitation is problematic, because it overshadows infants' agency. Similar problems pertain to the popular media phenomenon of "copycat babies". In different ways, both science and popular media tend to "other" infants: to portray them as radically different from "us" in ways that suggest inferiority (Powell \& Menendian, 2016).

As expressed in the Convention, the goals of education are the development of "the child's personality, talents and mental and physical ability to their fullest potential"; the development of respect for others, cultural values and the natural environment; and the preparation of the child for a "responsible life ... in the spirit of understanding" (29). I argue that we can best support these qualities if we treat the infant on equal terms: encountering the infant in ways one would like to be encountered, with respect, curiosity and understanding. To end, I suggest that if we look at infants acts in terms of play, recognising multimodality and improvisation, we can better understand their cultural agency, for example in co-creating communicative events.

\section{An Existential Approach}

Research on infants from the 197os onwards has radically changed the understanding of their cognitive and communicative abilities. Overall, the change has been from seeing young infants as mechanically responding creatures, driven by instincts and needs and not yet in control of their own movements, towards seeing infants as communicative persons who, while awake, are more or less constantly making sense of their surroundings and, although unfamiliar with cultural conventions, have already acquired some cultural learning. We know for instance that newborns can recognise auditory elements such as their mother tongue, their mother's voice, and music that they heard while in the womb (Stern, 2010; Moon, Lagercrantz, \& Kuhl, 2012; Huotilainen, 2012).

In the existential approach, I include research that emphasises infant's intersubjectivity, embodiment, (pro-) active behaviour, creative imagination 
and multimodal communication. Rather than separate, these aspects support and build on each other. The researchers that I include in this approach (see below) are sensitive to the question of what it is like to be a baby, and recognise the subjective character of infant experience and its irreducibility to mechanistic or objectifying explanations. In other words, while e.g., neurophysiology enhances our understanding of infants' embodied mind and its capacities, such knowledge cannot replace the question of what it is like to be (in) that body and situation. ${ }^{5}$

Infants' dependence on adult caregivers is undisputable. Yet the recognition of how much this dependence is social in character, rather than simply related to the fulfilment of physical needs, is more recent. The distinction between "primary" and "secondary" intersubjectivity (Trevarthen, 2001) suggests that the fundamental form of sociability - of being with and acting with others - does not presuppose a developed sense of self, i.e., self-awareness or self-consciousness (cf. Delafield-Butt, 2018). Rather, as suggested by Margaret Donaldson (1978) and Daniel Stern (1985/20oo, pp. 69-123), the infant who interacts with a parent is first a deictic and relational self who acts from its present situation without having internalised, and even less reflected upon, a selfimage, not to speak of self-identity. Being with others, rather than being alone or separate from others, is the default mode of infant existence and part of infant subjectivity. While infants are awake, especially in interactive situations, and often while they sleep, another person often holds them. The younger the infant, the more connected - to use Suzanne Zeedyk's key concept - it is, unable to change location and thereby enlarge its world to any significant degree without the help of others (Zeedyk, 2012). This does not mean, however, that infants are unable to separate self and other, only that their present and accumulated experience, including self-other relationships, differs significantly from adults' experience.

Colwyn Trevarthen (2011) describes exchanges between infant and parent as a "sharing of experiences". The formulation underlines that the experience is a joint creation that would not exist if either party were missing. It also indicates that experience is in the world, in a space between people, rather than just a mental occurrence. In a similar vein, Vasudevi Reddy criticises the idea of a "gap" between minds. In her "second-person perspective", infant and parent understand each other precisely through engagement and participation (Reddy, 2008, pp. 7-42). Maurice Merleau-Ponty (1945/1992) likewise pointed to the relevance of co-creating and sharing situations for understanding others. In these models, the emphasis is on connections within a dynamic, fluctuating exchange - rather than on the attempt to reach or identify the "content" 
of another mind, conceived as a static object. We understand each other in and through acting together partly because we change and grow precisely in these interactions, which the participants create jointly (Mühlhoff, 2015). ${ }^{6}$

Analyses of infants' early interactions indicate that the child is more than just reactive. Overall, there seems to be no reason to suppose a stage in infant development where the baby is unable to contribute in unexpected ways to social interactions, in however minute a manner. The premature baby that vocalised in response to the father's talk while kangarooing on his chest is an early example of infants' willingness and ability to have a conversation or join into what another person is doing. A recording showed that the infant contributed in meaningful, vocal ways to the father's talk, with exact timing (Trevarthen, 2018, p. 22).

Without recording, the premature infant's contribution to the conversation might have gone unnoticed. The communicative agency of young infants can indeed be hard to detect because it is low-key, but also because the recognition of the communicative intention of multimodal gestures might go unnoticed. Infants communicate and process things with their whole body more than adults do, and expressive gestures involve tensions and postures of the torso and movements of feet and legs in addition to hands and arms (Trevarthen, 2011). Mostly the eyes have a key role in communication, yet visual impairment is no hindrance to personal contact. There are many ways to turn towards or away from another and to signal contact or withdrawal. Moreover, the borderline between intended and spontaneous is probably open and permeable rather than fixed beforehand. When the other person recognises a gesture and responds, the gesture is more likely to become part of a cultural repertoire.

Overall, infants' communicative agency is embodied, multimodally gestural, and highly dialogic. The last feature is visible in their capacity for timing in socially interacting with an adult, often in situations where the focus is on enjoying the exchange itself. In a landmark book, Stephen Malloch and Colwyn Trevarthen (2009a) introduced "communicative musicality" to characterise infants' innate communicative capacity. Several features however invite us to use the term aesthetic here (cf. von Bonsdorff, 2018). First, the exchanges are multimodal rather than musical in the narrower sense of referring only to auditory qualities. Moreover, they are sensuous, expressive, intrinsically meaningful, (with form inseparable from content), ${ }^{7}$ and enjoyed for their own sake, ${ }^{8}$ for the fun of it. Finally, the infant's agency is - like the parent's - creative and imaginative: it is forward-looking and creates variations on earlier acts rather than mere repetitions. 
The cognitive approach differs in important respects from the existential, and is part of the legacy of Jean Piaget, whose primary focus was cognitive rather than social development. ${ }^{9}$ Cognitivists, such as Paul Harris (2000) and Alison Gopnik (2009), typically study infants and children with a view to rational thinking, and admire their capacity for understanding logical relationships such as causality and counterfactuals. Infants' and children's thinking is, in other words, assessed against criteria of logic, and explained with reference to mental maps and models. Moreover, children are studied as individuals, whose subjectivity is basically located in the brain. When it comes to understanding other persons, the idea is either that the infant simulates the other person's behaviour and likely feelings (simulation theory) or constructs a theory of the other's mind ("theory-theory"; see Reddy, 2008, p. 20). ${ }^{10}$ In other words, cognitivists presuppose that infants perform complicated operations of rational thinking and inference but lack any primary sense of intersubjectivity.

The particular cognitivist theory that I shall look upon in more detail is Andrew N. Meltzoff's explanation of responsive facial gestures in infants, a phenomenon usually referred to as imitation (Meltzoff, 2005). My aim is to indicate the limitations of the cognitive approach, as it sidesteps the potential social intentions of the infant and fails to account for its embodied and interactive situatedness (of by default being-with-another). Meltzoff's theory is cognitivist and mentalist, and even computational. On the one hand, it assumes that infants react to gestures due to innate mechanisms in the brain, while they on the other hand make rational comparisons ("if a, then p"). This is why the infant in imitation theory appears to be a computational baby.

Meltzoff observed that his new-born, only 42 minutes after birth, repeated the gesture of tongue protrusion that he had performed while looking his child in the eye (Meltzoff, 2005, p. 70). The fact that infants respond to an adult's facial gesture by repeating the same gesture, and may initiate the same gesture in subsequent situations with an adult, is now well recognised and widely researched by Meltzoff and others. Significantly, the phenomenon is discussed in terms of "imitation", and when initiated by the infant on a later occasion, "deferred imitation", which reportedly has happened after a delay of up to 24 hours (Meltzoff, 2005, p. 71).

According to Meltzoff, (2005, p. 56) imitation is innate, it precedes the understanding of other people, and these are causally related. He stipulates three conditions for imitation: "( 1 ) the observer produces behavior similar to that of the model, (2) the perception of an act causes the observer's response, and (3) the equivalence between the acts of self and other plays a role in generating 
the response"; adding however that the "equivalence need not be registered on a conscious level" (Meltzoff, 2005, p. 55). He also specifies that imitation takes place with novel acts and after a certain temporal delay, thereby distinguishing it from mere entrainment.

What does this mean in a situation of interaction between infants and adults? The first condition is unproblematic. The second condition however implies the causal activation of a particular mechanism. The infant does not make a choice, but is compelled to respond, and the reaction is purportedly due to the "imitative brain". Accordingly, Meltzoff's analysis is in terms of physiological rather than intentional activity. Imitation is a "matching-totarget-process" which includes the activation of exteroceptive and proprioceptive feedback loops. Infants identify relevant body parts and then attempt to perform the gesture they have perceived. A supramodal framework couples the observation and execution of acts, and enables the infants to repeat an act without knowing how their own face looks. This connection is innate: "exteroception (perception of others) and proprioception (perception of self) speak the same language from birth" (Meltzoff, 2005, p. 72).

The third condition of imitation, "the equivalence between the acts of self and other", is key in respect to Meltzoff's "Like-Me" hypothesis. When infants realise that the adult is like them, they acquire "a tool for cracking the problem of other minds." (Meltzoff, 2005, p. 75). Through coupling their own mental states (emotions, beliefs, intentions, etc.) to acts, and then registering the equivalence between self and other, they become able to understand the other. If I cry when I am unhappy, others are unhappy when they cry. Meltzoff assumes that there is a "lingua franca of human acts" (Meltzoff, 2005, pp. $75^{-76) \text {. }}$

As we have seen, Meltzoff's focus in analysing responsive acts is the act itself rather than its communicative or social intentions and functions, not to speak of context. His perspective is thoroughly third-person: there is no attempt to grasp intentions from a first-person perspective, nor to draw upon secondperson engagement in the situation (after all, the seminal insight came through interaction with his own child). This perspective is in harmony with the idea that the intersubjective element follows from the act, rather than being an ingredient of it.

In emphasising the developmental function of imitation for understanding other persons, Meltzoff restricts imitation to infants and demarcates it from adult life, where, as he writes, "certain bodily movements have particular meanings. If a person looks up into the sky, bystanders follow his or her gaze. This is not imitation; the adults are trying to see what the person is looking at" (Meltzoff, 2005, p. 65). Nevertheless, with a view to adult culture, if I feel 
that another imitates me, my reaction is likely to be either amused, baffled, or offended, depending on the situation. Not so if others respond to what I do: I then get a sense of mutuality, regardless of whether I think they got what I meant or not. Meltzoff also describes the function of imitation in infancy as learning a culture. However, the theoretical framework suggests that culture in imitation theory is more about socialisation and repetition than about variation and creativity. Overall, imitation theory pulls the understanding of infant agency in a hierarchical direction, where the adult is a model for the infant. The infant reacts, rather than acts, by imitating an act performed by an adult. However, the fact that the infant does not always respond has perhaps gained too little attention. While an infant would hardly perform a particular facial gesture without the adult performing it first, the adult's gestures may be a reason, but not a cause, for the infant's gesture. The distinction is important since it points to choice and intention.

There is an additional problem, related to the contingency of the facial gestures. Meltzoff claims that infants connect a particular gesture with their own "felt meaning", and then project this meaning on others who perform the same gesture. Nevertheless, the facial gestures used in the experiments may lack particular meaning in the culture, or the meaning they have may be irrelevant. To show one's tongue is a naughty, derogatory gesture in some cultures, while in others it signals a greeting. If new-borns protrude their tongue, they may just test how the air feels. There is no intrinsic "felt meaning" attached to the act - and whatever it might feel like probably has no communicative relevance (which Meltzoff also does not suggest).

Meltzoff can also be criticised for intellectualising the infant in three respects: in his privileging of vision, in the view of the infant self, and in the hypothesis thesis. Referring to how actions "look like" to the infant, or what a "new-born sees", he fails to notice how vision is imbricated in the total, multisensuous perceptual and embodied situation (Meltzoff, 2005, p. 75). ${ }^{11}$ To be born is to leave a mother's body, be received (mostly) by another adult, and then returned to intimate contact with mother for nursing and rest. When facial gestures are initiated, even if within one hour from birth, the newborn has already been addressed vocally, touched and held by adults. In these situations, people have probably looked the baby into the face, trying to catch attention and make contact. Adults as totalities with faces, gesturing and supportive bodies and vocalisations are familiar from the start of life. In these situations, interaction and communication in several sense modalities (sometimes tacit, sometimes more explicit) are paramount.

Through the multimodal and embodied immediate life-world, infants are connected to the world around, not separate. Infants live through and with 
other people. The multimodal and relational experience of the world makes the comparison between what is "seen" and "felt" dubious. Rather than reflection, implied by Meltzoff's phrasing, there is response from within a situation of primary connectedness. This connection is internal and runs like cords through the situation, rather than external, as when a cord binds two separate items. Furthermore, it is unlikely that infants experience their self or that of others in terms of mind-body -dualism. To separate "felt events" and "perceived events" presupposes a distinction of internal and external, but as argued above, the new-born self is neither articulate nor autonomous in a self-conscious way. More likely, in situations where facial gestures are initiated, new-borns recognise being addressed by another creature who, in a way that tickles their curiosity, is familiar and yet presents something new.

Finally, similar problems pertain to the idea that infants hold a hypothesis, however intuitive, about the similarity of self and other. This again presupposes an understanding or minimally a felt awareness of self and other as separate; but such an understanding probably does not yet exist. Although intelligent and exploring, new-borns hardly think in the scientific style. Rather than a hypothesis, there is a dawning felt sense, accompanied by experiential knowledge that grows in action. I shall elaborate on this in a later section, having first looked at some material from popular media.

\section{Copycat Babies}

Youtube searches for material on interactions with infants, using search terms such as "still-face experiment", "facial gestures babies" (or "infants"), "tongue protrusion" (with "babies" or "infants") or "facial gestures imitation infants" yield interesting results. I have made these searches at intervals from 2015 to 2019, and the material that comes up changes. Overall, however, there are three categories. First, some of the videos represent the popularisation of science for a larger audience, with interviews of renowned scientists. ${ }^{12}$ A second group comprises videos made for therapeutic or educational purposes, e.g. to help parents interact with their babies, support them in becoming more communicative and developing socially, or be more sensitive to the infant's gestures and intentions. Thirdly, there are homemade videos of infants' gestures, sometimes with interactions between infants and parents. These are the most interesting here. For ethical reasons I do not provide a list of the videos as it would be an unnecessary act of poking at behaviour that, while appearing in a less favourable light, hopefully was produced (by the adult/parent) without bad intentions. In addition, as intentionally recorded, the videos do not necessarily 
give a sense of what the everyday interactions between these infants and their parents are like.

There is naturally a rich variety in the videos but they also share some features. Often they focus on the infant's face and hands; sometimes they show the whole body. The babies are filmed in situations of active gesturing and vocalising, and appear "cute", "funny" or even "adorable". This is emphasised by the adult laughter that often accompanies the scene.

In one video, a mother is interacting with her baby vocally. The mutual vocalisations are clearly the focus of the video, although the infant, at two months, also makes lively gestures especially with her hands and arms. The mother is bent over and focusing on her child. She vocalises, and waits for the baby to respond, but then gives back more or less the same vocalisation, as if imitating or copying her child. Lying on her back, the baby looks confused at times; she frowns and looks away although she cannot escape the situation. On closer inspection, there seems to be many moments of standstill or interruption: the situation does not develop and the vocalisations remain separate. The mother's behaviour is enthusiastically forthcoming rather than attentive, and the baby is not fully "in" the situation either. One gets the sense that the performance is for the camera rather than a genuine, spontaneous dialogue. The mother is stimulating the baby to vocalise rather than interacting with her.

Some videos focus on tongue protrusion. In one of these, a mother is encouraging her child vocally and by touching tongue and mouth with a cloth. The act seems completely unnatural, and devoid of any context or meaning. In videos of babies performing "funny tongue movements", it is often not clear whether they respond to someone or not. The main purpose seems to be performing for amusement, but without the performer's (the infant's) consent or intention to amuse others.

In many of the videos, the infant's gestures are imitative in the colloquial sense: one person does something, and another repeats it. There is no need to understand context because the focus is simply on the quality of an act, not on the agent's intentions. This is the way imitation is performed in circuses or in contexts where the aim is mockery: to show someone's way of acting, make the audience aware of it, and laugh. Part of the videos seem to be set up for the camera, which raises the suspicion that the baby is trained to do the trick - this at least could be the case with many videos of tongue protrusion. There is a certain uncanny similarity to pet videos. Nevertheless, if the aim is to show what the infant is able to do, then laughter introduces a paternalistic perspective. Usually, we do not laugh at people acting smartly, but some laugh at people who misunderstand, make other mistakes, or act stupidly. From this perspective, the videos represent a practice of "othering", i.e. of representing 
the group of infants and toddlers as unlike us in ways that underline their inferiority (cf. Powell \& Menendian, 2016, p. 17). This raises a sense of uncanniness: why should we laugh, after all, and in what way?

To be fair, there are videos where parents seem to imitate their babies, or where imitation is ubiquitous. In one of the videos, a father, an infant and a dog inspire each other to howl. From a cultural perspective, we might assume that the funny actors are meant to be the dog and the infant rather than the adult. We laugh at them and with him, partly because adults constitute the "we-group" that set up the performance. "Adultism", or taking the adult as a norm while marginalising the child (Kennedy, 2006, p. 67), is probably at work here. Infants who imitate their parents are funnier than parents who imitate babies. The infant is the target, the object, not a subject.

In addition to mirroring general cultural norms and popular ideas, the videos seem to share certain characteristics with imitation theory, such as treating responsive acts and facial gestures separately from their larger context. Both imitation theory and the copycat videos combine a mechanical approach with a rationalising one: the baby as computer. In the videos, presenting infants' acts as funny underlines that the infant does not know what it does, but acts more or less mechanically in the way the adult does. Likewise, for imitation theory, the adult is the norm towards which the child strives. Finally, the videos and imitation theory seem to share an assumption of infants as less-than(adult)-humans, perhaps even not-yet-human.

\section{$5 \quad$ Play}

If imitation theory is problematic, how can we construe an alternative explanation of responsive acts? According to imitation theory, the infant first identifies relevant body parts (mouth and tongue) and then performs the act. However, if we approach the situation as a lived event, the reaction can be described as more immediate: responding by gesture to gesture. We move, and we move with others. According to Jonathan Delafield-Butt (2018, p. 59) the "two psychological principles that drive human agency" are "I like to move it" and "I like to move it with you." Perhaps responding to another's act is more like play than imitation?

How does the infant know its body and recognise similar other bodies? From a phenomenological perspective, the movements and acts of foetuses in the womb, exploring their own body, is at the same time a temporal and spatial structuring of the world. The nose, the hand and the toe are not visually seen but haptically and kinaesthetically explored. To modify Merleau-Ponty's 
(1992) observation of the two hands touching each other, with reciprocity and switch between which feels and which is felt: when foetuses or infants suck their thumb, attention can switch from the tongue exploring the thumb to the thumb exploring the mouth. Perceiving is multimodal: an exploration of forms and qualities that are recognisable through different sense modalities. Because the senses interact, a mouth that is seen as part of a face can be recognised based on earlier experiences of touching and feeling one's own face. There are the same forms, in the same constellation, doing similar things. Perhaps this is, or is at least part of, the supramodal framework Meltzoff (2005) assumes. Moreover, we can hardly overemphasise the importance of movement and rhythm in the life and development of foetuses and infants. ${ }^{13}$ What the infant recognises in the adult's figure is not just static forms but a dynamic constellation of form and movement: animated flesh similar to myself, acting like me.

Moreover, a facial gesture such as tongue protrusion is an intentional act, not a mechanical or necessary reflex. The infant-person has the option of not responding with a similar gesture - which does not mean no response at all. In a typical situation, the adult holds the infant and they focus on each other's faces where minute movements form an overall expressive and dynamic texture. In addition, felt muscular tensions are part of the overall directedness of one's own and the other's body, that connect haptically and kinaesthetically with each other. When adults perform a facial gesture and new-borns respond, the situation of being held and attending to another is familiar while the gesture is new and contingent. Precisely this novelty, and the opportunity to engage in interaction, may be what stimulates, or interests, the infant.

The contingency of the act implies that meaning arises in the situation. This is the case with greetings, which are precisely reciprocal exchanges of similar gestures, vocal or physical. A greeting is a fundamental way of acknowledging another person as a person. Exchanging variations of facial gestures likewise establishes and affirms mutual recognition and the constitution of a we (not any persons); a minimal sub-culture where participants can be together while being different. The exchanges comprise a way of acting and being (a manner or style) but they also demand attention and willingness to reciprocate, to play, on each side.

The interaction, as a play of mutual response, establishes "ways-of-beingwith" (Stern, 1985/200o, p. xv) construed by the two parties and established in the process (cf. Merleau-Ponty, 2003). Both of them learn. In my vignette, the newborn recognised me as the "tongue-showing one", and later took up the game with that particular person, not with others. I, the adult, had also learnt, in this case from research literature, that playing with the tongue is a possible form of interaction with infants. Instead of deferred imitation, suggesting 
mechanical causation, the infant's reason to initiate the game is probably curiosity, enjoyment, eagerness to establish a contact, or a mix of these - exactly like my own.

Playing with mutually responsive acts creates a tool for dialogue. Dialogue would not take place if the interaction were not a case of repeating differently, in other words a play that includes variation and improvisation. Infants are extremely sensitive to timing and nuances, and non-verbal dialogue is indeed about individual situations rather than generalised content, as suggested by the idea of comparing "felt event" and "seen event". Instead, the interactions are similar to arts of performance such as music or dance, especially when they involve improvisation and dialogue (cf. Stern, 2010). They are foundational for culture because they include freedom, choice, and variation, rather than mere repetition or imitation. The impossibility of strict imitation is a resource for communication, not a limitation.

A contingent gesture, which serves no particular function nor conveys any particular meaning, is a seed of shared aesthetic and intellectual explorations. This is precisely because it initially has no connection to physical needs and predetermined meaning. Rather, the exchange is performed for enjoyment, and for its own sake alone. ${ }^{14}$ Mutual tongue protrusion, or other gestural exchanges that do not serve a particular goal, give rise to a new kind of intersubjectivity. The other is no longer just the one who takes care of me or the one who is taken care of by me, but someone interested in playing with me. The exchange of contingent gestures therefore opens a new and exciting dimension of subjectivity, related to the recognition of the other as another person, and a cultural subject.

Already in situations of early exchange, infants are capable of being culturally inventive, as my vignette shows. Without the initiative of the infant girl, our initial tongue protrusion might have remained a single occurrence, but she established it as a shared practice.

In the Introduction, I suggested a lacuna in the Convention when it comes to interaction and communication, which makes it hard to see how we can provide for infants' cultural rights. The cognitivist explanation of responsive acts does not help, as it largely misses the improvisational character of early interactions, where adjustments to the other person go on all the time. There is a serious limitation regarding infant subjectivity and intersubjectivity, and how they develop in interactions. The importance of this reaches beyond the 
academic world, for theories of infant development interest parents. Perhaps not surprisingly, the phenomenon of copycat babies show uncanny similarities to current cognitivist theories. While seemingly demonstrating what infants can do, they in fact often show situations with one-way, disconnected utterances. They maintain a gap between adult and infant, and between embodied gesture and mind.

Our beliefs of what infants can do and how they think influence our interactions with them. We treat them differently if we assume, they are only capable of imitation, as compared to how we treat them when we think of them as persons with interests and intentions. A strength of the existential approach is that it sees the infant as an individual in a certain situation, and does not take for granted categorical differences, for example in terms of intentionality, between infant and adult. The theories I have presented above as part of the existential approach also strongly acknowledge intersubjectivity, in fact in ways that challenge the traditional western ideal of a rational, autonomous subject.

The Convention's goals of education refer to the development the child's personality, in the spirit of respect and responsibility (29). We can only attain these goals if we treat the child in a like manner. Reciprocal, playful practices may be a critical ingredient of the ethical goals of education. Shared meaning, but also self and world are established and modified in these practices. Moreover, both parties are affected. The willingness to give of oneself and receive from the other is a key to human growth and culture. To get a mechanical response, we need not be fully attentive to the other, whereas play demands sensitivity and presence on both sides. An existential approach provides us with models and tools that can help understanding not just the infant, but also the adult of the relationship in more reflective ways.

\section{Notes}

1 This incident took place in October 2016 in Copenhagen.

2 From now on, I refer to it as the Convention. Reference numbers are to the Articles of the Convention.

3 The Convention includes one article (23) on disabled children - emphasising dignity, selfreliance and active participation - but this does not change the overall picture.

4 It also states "the right of the child to rest and leisure, to engage in play and recreational activities appropriate to the age of the child and to participate freely in cultural life and the arts" (article 31:1).

5 For a classical but still pertinent discussion of the limits of understanding what it is to be like someone radically different from oneself, see Nagel (1974). 
6 This structure differs from the parallel minds that would come into play in "joint attention". However, to attend to something in the company of other people typically involves minute reactions that are perceptible to others. Even at a concert of classical music, where audience response is minimal, we are aware of the reactions of people in our vicinity, and especially of our companions.

7 Alloa and Jdey (2012) point out that for Merleau-Ponty, the body resembles a work of art exactly in this way. Relevant here is also Waldenfels' (2015, pp. 133-158) discussion of the aesthetic in Merleau-Ponty.

8 See Reddy's (2008, pp. 183-214) discussion of the sense of humour, or "funniness", in young children.

9 As studied by Piaget, the young child is an individual, even "egocentric", has no moral concepts and is unable to distinguish fantasy from reality. Development takes place through separate stages. For critical assessments of Piaget, see Merleau-Ponty (2001), Donaldson (1978), Trevarthen (2001), Reddy (2008). For critiques of cognitivism, see Reddy (2008, pp. 7-25) and Stock (2011).

10 Reddy (2008) and Trevarthen (2007, p. 12) both remind that the "theory of mind" is culture relative, not universal.

11 On the same page he writes: "Human acts are especially relevant to infants because they look like the infant feels himself to be and because they are events that infants can intend. When a new-born sees a human act, it may be meaningful: "That seen event is like this felt event" (hyphenation added). More recently, Meltzoff has studied touch and emphasised its importance for the body scheme and for social cognition. But to my knowledge, he has not revised the theory of imitation with a view to these themes.

12 Tongue protrusion as typical of Down's syndrome also comes up, but I shall put that aside.

13 Stern (2010) points out that the foetus moves before it has a brain, and Delafield-Butt (2018) suggests that there is a "brainstem self" with "basic self-consciousness" from 12-14 weeks of gestation.

14 The hallmark of the aesthetic, as traditionally defined, is precisely that its value is intrinsic and serves no exterior ends. It has also been described as "autotelic": having its end in itself.

\section{References}

Alloa, E., \& Jdey, A. (2012). Du sensible à l'oeuvre. Sur le rapport entre Merleau-Ponty et les arts. In E. Alloa \& A. Jdey (Eds.), Du sensible à l'oeuvre. Esthétiques de MerleauPonty (pp. 9-39). La Lettre volée.

Corsaro, W. A. (2018). The sociology of childhood (5th ed.). Sage Publications.

Delafield-Butt, J. (2018). "The emotional and embodied nature of human understanding: Sharing narratives of meaning." In J. Delafield-Butt, A.-W. Dunlop, \& C. Trevarthen (Eds.), The child's curriculum: Working with the natural values of young children (pp. 59-84). Oxford University Press.

Donaldson, M. (1978). Children's minds. Fontana/Collins.

Gopnik, A. (2009). The philosophical baby. What children's minds tell us about truth, love, and the meaning of life. Farrar, Straus \& Giroux.

Harris, P. L. (2000). The work of the imagination. Blackwell. 
Huotilainen, M. (2012). "A new dimension on Foetal language learning". Acta Paediatrica, 102(2), 102-103. https://doi.org/10.1111/apa.12122

James, A., Jenks, C., \& Prout, A. (1998). Theorizing childhood. Polity Press.

Kennedy, D. (2006). The well of being. Childhood, subjectivity, and education. State University of New York Press.

Malloch, S., \& Trevarthen, C. (Eds.). (20oga). Communicative musicality. Exploring the basis of human companionship. Oxford University Press.

Malloch, S., \& Trevarthen, C. (2009b). Musicality: Communicating the vitality and interests of life. In S. Malloch \& C. Trevarthen (Eds.), Communicative musicality. Exploring the basis of human companionship (pp.1-11). Oxford University Press.

Meltzoff, A. N. (2005). Imitation and other minds. The "like me" hypothesis. In S. Hurley \& N. Chater (Eds.), Perspectives on imitation: From neuroscience to social science (Vol.2, pp. 55-77). The MIT Press.

Merleau-Ponty, M. (1992). Phénoménologie de la perception. Gallimard. (Original work published 1945)

Merleau-Ponty, M. (2001). Psychologie et pédogogie de l'enfant. Cours de Sorbonne 1949-1952. Verdier.

Merleau-Ponty, M. (2003). L'Institution. La Passivité. Notes de cours au Collège de France (1954-1955). Belin.

Moon, C., Lagercrantz, H., \& Kuhl, P. K. (2012). "Language experienced in utero affects vowel perception after birth: A two-country study”. Acta Paediatrica, 102(2), 156-16o. https://doi.org/10.1111/apa.12098.

Mühlhoff, R. (2015). Affective resonance and social interaction. Phenomenology and the Cognitive Sciences, 14, 1001-1019.

Nagel, T. (1974). "What is it like to be a bat?" The Philosophical Review, 83(4), 435-450.

Powell, J. A., \& Menendian, S. (2016). The problem of othering: Towards inclusiveness and belonging. Othering \& Belonging. Expanding the Circle of Human Concern, 1, 14-39. Retrieved September 15, 2019, from http://www.otheringandbelonging.org/ the-problem-of-othering/

Reddy, V. (2008). How children know minds. Harvard University Press.

Stern, D. N. (2000). The interpersonal world of the infant. A view from psychoanalysis and developmental psychology. Basic Books. (Original work published 1985)

Stern, D. N. (2010). Forms of vitality. Exploring dynamic experience in psychology, the arts, psychotherapy, and development. Oxford University Press.

Stock, K. (2011). Unpacking the boxes. The cognitive theory of imagination and aesthetics. In E. Schellekens \& P. Goldie (Eds.), The aesthetic mind. Philosophy and psychology (pp. 268-282). Oxford University Press.

Trevarthen, C. (2001). The neurobiology of early communication: Intersubjective regulations in human brain development. In A. F. Kalverboer \& A. Gramsbergen (Eds.), Handbook on brain and behavior in human development (pp. 841-882). Kluwer. 
Trevarthen, C. (2007). Wer schreibt die Autobiographie eines Kindes? In H. Welzer \& H. J. Markowitsch (Eds.), Warum Menschen sich erinnern können. Fortschritte der interdisziplinären Gedächtnisforschung (pp. 225-255). Klett-Cott.

Trevarthen, C. (2011). The generation of human meaning: How shared experience grows in infancy. In A. Seemann (Ed.), Joint attention. New developments in psychology, philosophy of mind, and social neuroscience (pp. 73-114). The MIT Press.

Trevarthen, C. (2018). What young children give to our learning. In J. Delafield-Butt, A.-W. Dunlop, \& C. Trevarthen (Eds.), The child's curriculum: Working with the natural values of young children (pp. 13-38). Oxford University Press.

UNCRC. (1989). United Nations Convention on the Right of the Child. Retrieved April 17, 2020, from https://downloads.unicef.org.uk/wp-content/uploads/2010/o5/UNCRC_ united_nations_convention_on_the_rights_of_the_child.pdf? ga $=2.2595^{82415} \cdot 454887985 \cdot 1587459175-44770236.1585716747$

von Bonsdorff, P. (2018). Children's aesthetic agency: The pleasures and power of imagination. In J. Delafield-Butt, A.-W. Dunlop, \& C. Trevarthen (Eds.), The child's curriculum: Working with the natural values of young children (pp. 126-138). Oxford University Press.

Waldenfels, B. (2015). Sinne und Künste in Wechselspiel. Modi ästhetischer Erfahrung. Suhrkamp.

Zeedyk, M. S. (2012). Wired for communication. How neuroscience of infancy helps understanding the effectiveness of intensive interaction. In D. Hewett (Ed.), Interaction. Theoretical perspectives (pp. 55-71). Sage. 